\title{
A 2-substituted 8-hydroxyquinoline stimulates neural stem cell proliferation by modulating ROS signalling
}

Cathryn L. Haigh, ${ }^{1} *$ Carolin Tumpach, ${ }^{2}$ Steven J. Collins, ${ }^{1}$ and Simon C. Drew ${ }^{2, *}$

${ }^{1}$ Department of Medicine, Royal Melbourne Hospital, The University of Melbourne, Victoria 3010, Australia, ${ }^{2}$ The Florey Department of Neuroscience and Mental Health, The University of Melbourne, Victoria 3010, Australia.

* To whom correspondence should be addressed: sdrew@unimelb.edu.au (+61 39035 8684), chaigh@unimelb.edu.au (+61 38344 1952)

\section{Running Title:}

8HQ stimulation of NSC proliferation

\section{Keywords:}

8-hydroxyquinoline, neural stem cells, neurosphere, proliferation, NADPH oxidase, reactive oxygen species, 2-[(dimethylamino)methyl]-8-hydroxyquinoline, DMAMQ, PBT2 


\begin{abstract}
Eight-hydroxyquinolines (8HQs) are a class of compounds that have been identified as potential therapeutics for a number of neurodegenerative diseases. Understanding the influence of structural modifications to the 8HQ scaffold on cellular behaviour will aid the identification of compounds that might be effective in treating dementias. In this study, we describe the action of 2-[(dimethylamino)methyl]-8-hydroxyquinoline (DMAMQ) on adult murine neural stem cells (NSCs) cultured in vitro. Treatment of NSCs with DMAMQ resulted in enhanced self-renewal and increased neurite outgrowth. Concurrent with the positive growth effects was an increase in intra-cellular reactive oxygen species, with the growth being inhibited by inactivation of the NADPH oxidase (Nox) enzyme family. Our results indicate that DMAMQ can stimulate neurogenesis via the Nox signalling pathway, which may provide therapeutic benefit in treating dementias of various types by replenishing neurones using the brain's own reserves. The narrow concentration range over which these effects were observed, however, suggests there may exist only a small therapeutic window for neuro-regenerative applications.
\end{abstract}




\section{Introduction}

Endogenous neural stem cells (NSCs) are an as-yet untapped resource in combatting dementia. Low levels of NSCs persist in the brain throughout adult life, maintaining the ability to self-replicate and to differentiate into mature brain cells. During dementia, neurogenesis (the ability to form new neurones) is seen to become dysregulated $[1,2]$. In rodent models of Alzheimer's disease $(\mathrm{AD})$, production of beta-amyloid, one hallmark of $\mathrm{AD}$, changes the growth and differentiation of these cells $[3,4,5,6,7]$. Neurogenesis has been extensively linked with both learning and forgetting $[8,9,10]$; therefore, changes in these processes might contribute significantly to the cognitive changes that occur during neurodegenerative diseases, including AD. Compounds that modulate or normalise the functions of NSCs represent a prime target for alleviating the symptoms associated with, or delaying the course of, neurodegenerative diseases.

Unbiased chemical screening of substituted 8-hydroxyquinolines (8HQs) and a range of investigations in yeast, nematodes, mice and humans have highlighted the variable mechanisms by they may act. Side-chain modifications to the 8HQ backbone may lead to functional differences in vivo, with applications of substituted 8HQs as anti-microbial agents $[11,12]$, anti-cancer agents $[13,14]$, epigenetic modulators $[15,16]$, dementia treatments $[17,18,19,20]$, artificial nucleobases [21] and medical imaging agents [22,23]. Moreover, biosynthesis of substituted 8HQs has been identified in mammals (eg. xanthurenic acid [24]), bacteria (eg. quinolobactin [25]) and insects (eg. 2-carboxy-8HQ [26]). In dementia applications, studies utilising 5,7-dichloro-2-[(dimethylamino)methyl]-8-hydroxyquinoline reported an increased neurite number following treatment of cultured PC12 cells and a restoration of hippocampal dendritic spine density (but not number) in transgenic mouse model of $\mathrm{AD}[18]$. 
In the current study, we sought to further understand the action of this class of 8HQ by treating adult murine NSC cultures with 2-[(dimethylamino)methyl]-8-hydroxyquinoline (DMAMQ; Figure 1). We specifically assessed the ability of these cells to self-renew, essential for maintaining their numbers in the brain throughout life, and to differentiate into new CNS lineage cells. Our findings show that whilst neurogenesis is unchanged, NSCs treated with DMAMQ demonstrate a dose-dependent increase in NSC proliferation and increased neurite outgrowth during neurogenesis that was signalled by increased production of reactive oxygen species (ROS) signalling intermediates by the NADPH oxidase (Nox) enzyme family. These effects were only observed within a narrow concentration range, suggesting a small therapeutic window for neuro-regenerative applications. 


\section{Materials and Methods:}

Synthesis

2-[(dimethylamino)methyl]-8-hydroxyquinoline (DMAMQ) was synthesised as described previously [27].

\section{Cell culture}

Murine NSCs were harvested from the brains of 6-8 week old Balb/c mice and grown without modification as neurospheres in liquid culture as described previously [28].

\section{DMAMQ treatments}

DMAMQ was included in the culture medium at the concentrations indicated. For all assays, the compound was added as a single treatment at the start of the assay and was not replenished when the media was changed.

Neural Colony Forming Assay (NCFA)

The NCFA has been described previously [29]. For each independent experiment over 50 colonies were measured. As stated above, DMAMQ was included in the matrix once only at the start of the incubation.

Neurite outgrowth assay

Neurite outgrowth was measured using a neurite outgrowth staining kit (Merck-Millipore) as per the manufacturer's instructions with the following modifications. One hundred thousand cells per condition were incubated in high FGF (20 ng/mL), no EGF, growth media before 
seeding 100,000 cells per well insert $(1 \mu \mathrm{m}$ pore size $)$ in differentiation medium with or without test compound. Neurite outgrowth was permitted for two days before assay.

\section{Dichlorofluorescein (DCF) intracellular ROS assay}

The DCF assay has been described previously [30]. Cells were seeded at a density of $3.6 \times$ $10^{4}$ cells/well 24 hours before the start of the assay in poly-D lysine coated 96-well plates. Fluorescence intensity was measured every 5 min for 12 hours using a FLUOstar Optima (BMG Labtech) fitted with $490 \mathrm{~nm}$ excitation and $520 \mathrm{~nm}$ emission filters and initial rates were calculated using tangents to the curve.

\section{MTS metabolism assay}

Five $\mu \mathrm{L}$ of One Solution MTS reagent (Promega) per $100 \mu \mathrm{L}$ media was added to test and media only background control conditions, and incubated under normal culture conditions for $90 \mathrm{~min}$. Reaction product was quantified using absorbance at $462 \mathrm{~nm}$ in a FLUOstar Optima.

\section{$\beta$-galactosidase staining and quantification}

The cells were stained using a Cell Signaling Technologies' $\beta$-galactosidase staining kit as per the manufacturer's instructions. To solubilise the stain for quantitation by spectrometry, the staining solution was removed from the wells and replaced with $350 \mu \mathrm{L}$ of DMSO. The plate was warmed with agitation at $50^{\circ} \mathrm{C}$ for 1 hour before $100 \mu \mathrm{L}$ of dissolved dye was transferred to three wells of a 96-well plate for triplicate reads.

\section{Immunofluorescence staining}

The immunofluorescence staining protocol for NSC lineage has been described previously [31]. 
Ki67 expression in proliferating cells was quantified using the Muse Ki67 Proliferation Kit (Millipore) as per the manufacturer's instructions. For the final analysis the number of unstained cells gated as positive was subtracted from the stained cells and percentage change was calculated from the control cells.

\section{Cell cycle phase flow cytometry}

Cell cycle phase analysis by DNA content was determined using the Muse Cell Cycle Analysis Kit (Millipore) as per the manufacturer's instructions.

\section{PAGE and Western Blotting}

Polyacryalmide gel electrophoresis and western blotting was conducted as described previously [32]. Primary antibodies and concentrations were as follows; pMEK1, pMEK2 and pGSK-3ß (Abcam) 1 in 1000; nestin (Sigma) 1 in 1000; GFAP (Stem Cell Technologies) 1 in 5000; and Neurofilament-L (Invitrogen) 1 in 1000. Relative densitometric comparisons were normalised for total protein loading as shown in Coomassie stained images.

\section{Calcium assay}

Cells were seeded in 96-well plates as above. At the beginning of the assay $50 \mu 1$ of culture media was removed from each well and replaced with calcium assay working buffer as per manufacturer's instructions (Invitrogen). Plates were incubated for one hour under normal incubator conditions protected from light before test reagent addition. Readings were taken using $488 \mathrm{~nm}$ excitation and $530 \mathrm{~nm}$ emission filters in a FluoSTAR Optima (BMG Labtech) every 60 seconds for 1 hour from addition.

\section{Statistical analyses}


Statistical analyses were carried out using GraphPad Prism 5 statistical software. The relevant statistical test applied is indicated in the figure legend. Where significant differences were found, Dunnett, Bonferroni and Dunn's tests were used for multiple comparisons of oneway, two-way and non-parametric ANOVA, respectively. Graphs show the mean and standard error of the mean (SEM) of $n$ independent experiments unless otherwise stated. 


\section{Results and Discussion}

Herein we investigated the potential for DMAMQ to enhance the regenerative capacity of NSCs cultured in vitro. NSC cultures were established using cells harvested from the subventricular zone of adult wild type Balb/c mice and grown in suspension culture as neurospheres. We first examined the influence of DMAMQ on proliferating neurospheres using a colony forming assay. Cells were exposed to one single treatment included in the media-matrix at the start of the assay. Exposure of neurospheres to DMAMQ was observed to have a profound effect on the morphology (Figure 2a) and volume (Figure 2b) of the growing neurospheres. Significant toxicity of DMAMQ was only observed at the highest concentration tested (100 $\mu \mathrm{M}$; Figure S1), whereas NSC growth was stimulated in the low $\mu \mathrm{M}$ concentration range. The observed proliferative effect was due to both the number of new neurospheres/colonies being increased (Figure 2c) and the distribution of neurosphere sizes shifting towards larger diameters (Figure 2d). Since the greatest proliferative effect was observed at 2.5 $\mu \mathrm{M}$ DMAMQ, this concentration was used for all subsequent assays.

To investigate the increased growth further Ki67, a marker expressed only in actively proliferating cells, was assessed by flow cytometry. An approximate $4 \%$ increase in proliferating cells was seen after 24 hours of exposure to DMAMQ in normal culture media (Figure 3a,b). Cells that are not proliferating may be quiescent (resting in G0 until stimulated to re-enter the cell cycle) or senescent (an irrecoverable G0 state where cells are unable to reenter the cell cycle but are not dead). $\beta$-galactosidase staining is characteristic of senescent cells. Following 3 days of DMAMQ treatment, administered as a single dose, the number of senescent cells in the culture was decreased by almost $20 \%$ (Figure 3c). Analysis of cell cycle phase by DNA content also showed that cells were cycling more rapidly following DMAMQ treatment, with a lower percentage of cells resting in the G0 and G1 phases of the 
cell cycle following 24 hours DMAMQ treatment (Figure 3d,e). These results indicate that the observed increase in NSC proliferation was due to a shift towards active proliferation coupled with protection against senescence.

In addition to heightening the ability of NSCs to proliferate in the colony forming assay, we observed a distinct pattern of outgrowth from NSCs cultured in response to DMAMQ, suggesting that DMAMQ also promoted neurite outgrowth in the proliferating cultures (Figure 2a). To ascertain whether this was a significant event, the number of neurospheres demonstrating this morphology were counted and showed increased frequency in the DMAMQ treated colonies (Figure 3f). The ability of DMAMQ to stimulate neurite outgrowth during differentiation was assessed using a colourimetric neurite staining protocol and showed that, over two days of differentiation, DMAMQ-treated cells displayed ca. $10 \%$ more neurite outgrowth than was observed for control cells (Figure 3g).

To ascertain whether enhanced neurite outgrowth could be linked with enhanced neurogenesis (the capacity to form new neurones), the influence of DMAMQ on neuronal or astrocytic lineage preference following differentiation of NSCs was considered. Lineage commitment of NSCs can be influenced by factors both when stimulated to differentiate and before they are stimulated to differentiate. Therefore, we considered the effect of including DMAMQ in the culture media during differentiation and also before cells were differentiated as a 'priming' event. For the differentiating culture, DMAMQ was added at the induction of differentiation and remained in the media for 7 days. For the primed culture, NSCs were treated for 24 hours prior to differentiation and then transferred into differentiation medium and cultured for 7 days the absence of further exposure to DMAMQ. Subsequent immunofluorescence staining indicated that DMAMQ incubation did not alter the resulting cell lineages or overtly change the morphology of the differentiated neurones and astrocytes (Figure 4a). In addition, 
western blot analysis (Figure 4b) confirmed no changes in expression of nestin (Figure 4c), neurofilament-L (NF-L; Figure 4d) or GFAP (Figure 2e) either when cells were primed or differentiated with DMAMQ. Therefore, neurogenic potential of NSCs treated with DMAMQ was neither enhanced nor compromised.

Inactivation (by phosphorylation of Ser9) or pharmacological inhibition of GSK-3 leads to both increased proliferation and enhanced neurogenesis in neural stem cell cultures [33,34] and this phosphorylation has previously been reported in some studies using the 5,7dichlorinated analogue of DMAMQ [18]; however, we did not observe any change in the phosphorylation status of GSK-3 $\beta$ following treatment with concentrations of DMAMQ that induced NSC growth (Figure 5a). Indeed, a significant increase in phosphorylation was only observed at $100 \mu \mathrm{M}$ DMAMQ (Figure S2), the same concentration at which DMAMQ cytotoxicity was significant (Figure S1). Therefore, signalling through GSK-3 $\beta$ is unlikely to be of relevance to DMAMQ-stimulated NSC growth. Additionally, no detectable changes in AKT and MEK1/2 phosphorylation were observed (Figure 5a), indicating these redoxsensitive signalling pathways were not involved in the induction of cell growth.

Many neuronal signalling processes involve calcium flux and stem cells utilise these pathways [35]. Changes in calcium mobilisation are also linked with in redox signalling [36] and increased cytosolic $\mathrm{Ca}^{2+}$ can directly activate some Nox enzymes [37]. Therefore, intracellular calcium levels were measured in the NSCs. Following DMAMQ treatment, a reduction in intracellular calcium was detected (Figure 5b).

Redox signalling pathways have been linked with NSC growth and an increased ROS burst has been linked with induction of proliferation $[38,39,40]$. A 'cellular redox cycle' has been shown to occur within the cell cycle and is thought to control cycle phase progression $[41,42]$. Measurement of intracellular ROS production using the fluorescent probe DCFDA showed 
increased ROS production when NSCs were treated with DMAMQ in the micromolar range (Figure 6a). There are various sources of intracellular ROS including the Nox family of signalling proteins. The Nox family, which generates ROS as a signalling intermediate, has been linked with stimulation of NSC proliferation $[43,44,45]$. To examine the role of Nox signalling in the ROS produced in response to DMAMQ, a Nox isozyme inhibitor (DPI) was co-administered with DMAMQ in the DCFDA assay. Inclusion of DPI was sufficient to block the DMAMQ-induced ROS response in the NSCs (Figure 6b), implicating Nox in the observed intracellular ROS burst.

If the ROS produced by the addition of DMAMQ to the cells was signalling the increased growth of the cells, then inhibition of this pathway with the DPI Nox inhibitor would be expected to inhibit the increased colony growth in the NCFA. DPI was included in the media matrix as a single dose at the start of the NCFA with or without NNAMQ and growth was determined as previously. DPI alone reduced colony growth, primarily affecting the number of colonies formed (Figure 7a-c). When DPI and DMAMQ were co-incubated in the mediamatrix (single treatment at the start of the assay) the growth of the spheres was stunted, with fewer colonies formed (Figure 7a-c) and decreased diameter at the $2.5 \mu \mathrm{M}$ treatment concentrations of DMAMQ.

Whilst our results concur with previous literature that increased ROS increases NSC growth and suggest that modulation of Nox to increase ROS signalling initiates such growth, the data presented cannot exclude intrinsic ROS production by DMAMQ that mimics, but does not outcompete inhibition of, this pathway. In any case, the generation of ROS in response to DMAMQ treatment contrasts with the mainstream view that 8HQs can be of therapeutic benefit by reducing aberrant metal-mediated ROS production associated with neurodegenerative disease [46]. 
The identification of compounds that regulate neurogenesis holds great promise for treating neurodegenerative conditions. $\mathrm{AD}$-associated $\mathrm{A} \beta$ peptides have been shown to decrease neurogenesis using both in vivo and in vitro models, including murine NSC cultures $[3,47,48]$ and hence restoration of NSC function by $8 \mathrm{HQ}$ s could be of value in treating AD. The picture is far from clear, however, since markers of increased neurogenesis can be found in postmortem analysis of $\mathrm{AD}$ brains [2] and this is also found in some animal models of disease $[4,49]$. Increased neurogenesis may represent a compensatory response by the NSCs within the brain to replenish lost neurones. In such a context, the current findings suggest that compounds that stimulate neurogenesis during $\mathrm{AD}$ may be able to augment these compensatory mechanisms. The caveat, however, is that neurogenesis has also been linked with forgetting [8]; in theory, stimulating neurogenesis may therefore inadvertently exacerbate the symptoms of memory loss. In addition to these complications, which may arise even from a targeted stimulation of neurogenesis, Nox signalling regulates diverse processes in vivo such as contractile functions of heart muscle [50,51] and systemic immune responses [37,52], while acute and chronic Nox activity is associated with neuroinflammation [53]. Moreover, substituted $8 \mathrm{HQs}$ can have anti-proliferative/cytotoxic properties depending on their local concentrations (Figure S1) $[13,14,15]$ or have the potential to cause neuronal damage by interfering with epigenetic regulation (eg. clioquinol [54]). Some of these considerations may explain why clioquinol and 5,7-dichoro-DMAMQ have not shown evidence of clinical benefit in treating $\mathrm{AD}$ [55].

\section{Conclusions}

This study demonstrates that DMAMQ modulates NSC growth and neurite outgrowth during differentiation when cultured in vitro by stimulating ROS production and Nox signalling. 
This suggests that DMAMQ and similar 8HQs may have neuro-regenerative potential in vivo; however, the very narrow concentration range in which DMAMQ modulates NSC growth presents some challenges for therapeutic administration. In particular, the potential benefits of replenishing damaged brain tissue must be balanced against the possibility of overstimulating neurogenesis and the propensity for undesirable "off-target" effects. A substantial increase in our understanding of the action of substituted $8 \mathrm{HQs}$ in vitro and in vivo is required to determine whether this class of compound will be a viable therapeutic intervention in countering disease progression in any dementia.

\section{Acknowledgements}

This work was supported by a Future Fellowship (FT110100199) administered by the Australian Research Council (S.C.D.), a Program Grant (\#628946; S.J.C.) and Practitioner Fellowship (APP1005816; S.J.C.) administered by the National Health and Medical Research Council (NHMRC) of Australia. 


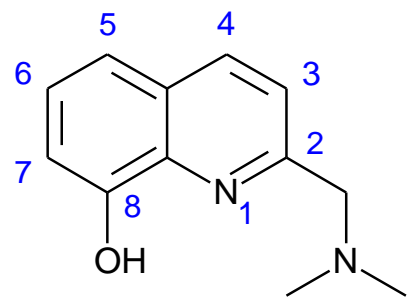

Figure 1. Structure of DMAMQ (2-[(dimethylamino)methyl]-8-hydroxyquinoline). 
(a)

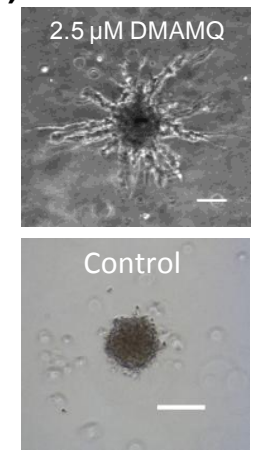

(b)

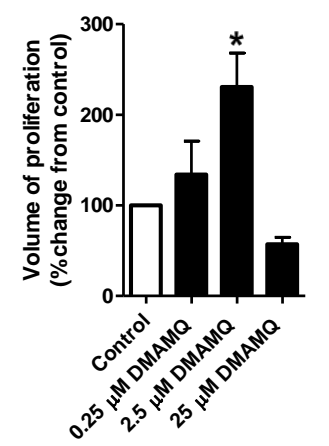

(c)

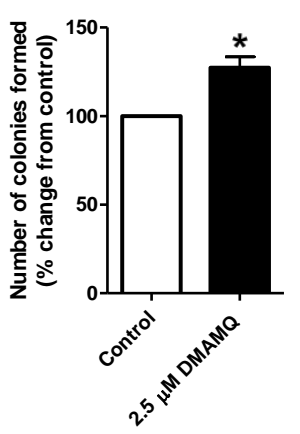

(d)

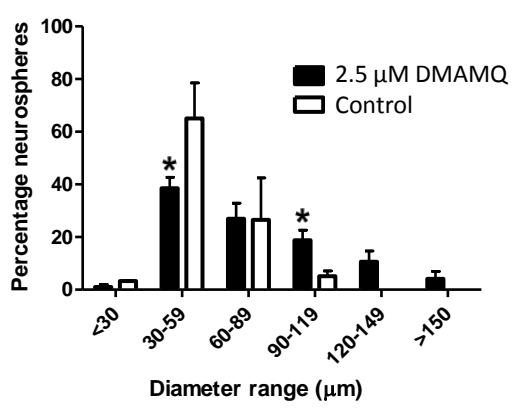

Figure 2. DMAMQ promotes the proliferation of NSCs. (a) Representative field of view showing neurosphere morphology with and without $2.5 \mu \mathrm{M}$ DMAMQ treatment for 21 days. Scale bar $=35 \mu \mathrm{m}$. (b) Colony volume measurement of increasing DMAMQ doses. Dissociated cells (single cells) were seeded at equal density with/without DMAMQ and the growth of neurospheres present at 21 days post plating measured (change in volume is expressed relative to the no DMAMQ control; one-way ANOVA, $n=3$ ). Neurosphere volume at $2.5 \mu \mathrm{M}$ DMAMQ is increased both because of (c) increased colony formation (Students $t$-test, $n=3$ ) and (d) a shift in the size of the neurospheres towards larger diameters (with significant differences being obtained for diameters in the 30-59 $\mu \mathrm{m}$ and $90-199 \mu \mathrm{m}$ range; two-way ANOVA, $p<0.001, n=3$ ). 
(a)
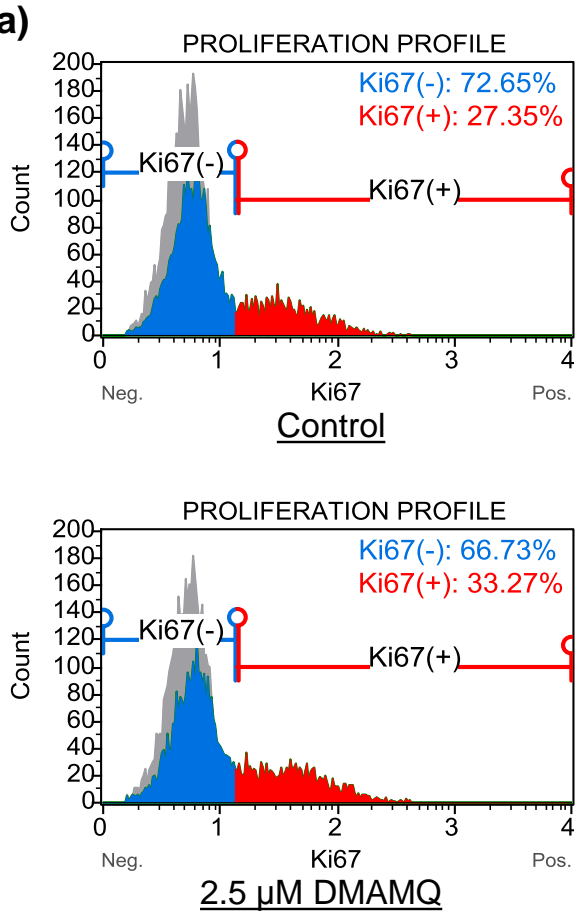

(e)

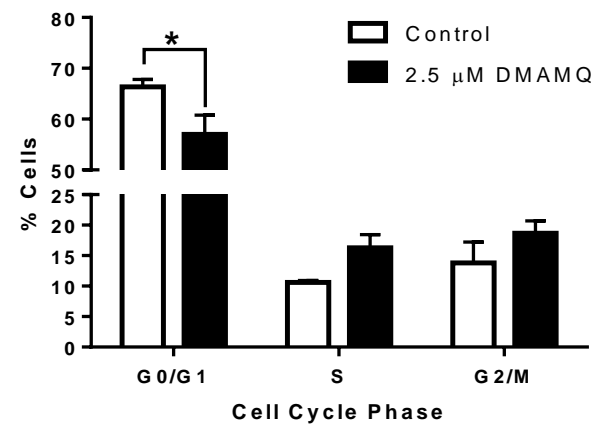

(b)

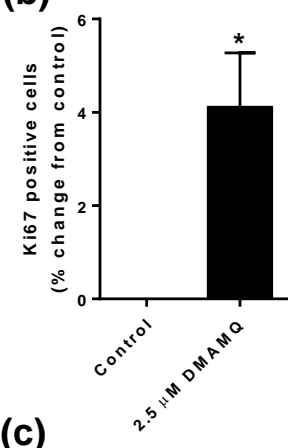

(c)

(d)
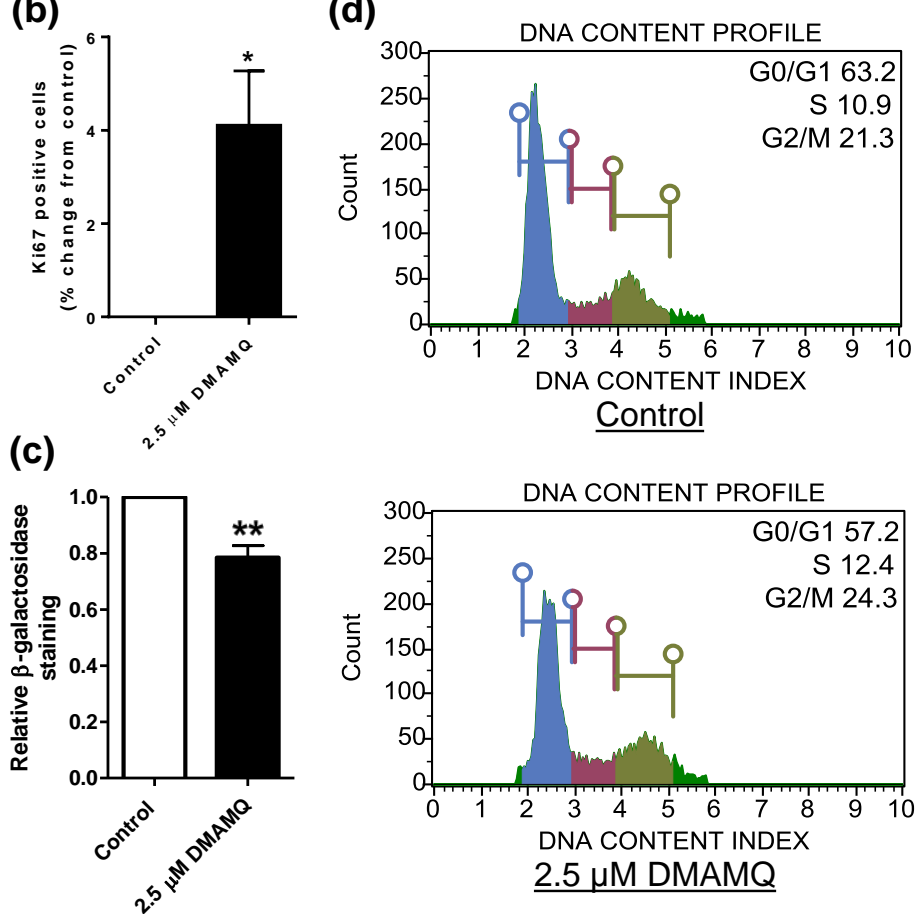

(g)

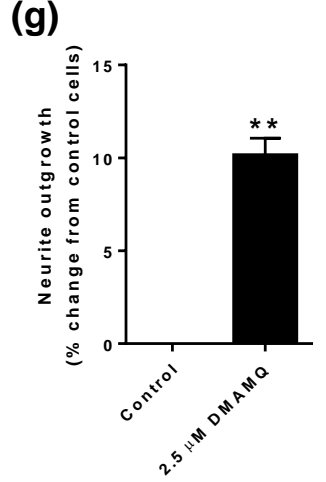

Figure 3. DMAMQ reduces the non-proliferative population of NSCs and increases NSC growth. (a) Flow cytometry analysis of Ki67 proliferation marker expression in NSCs after 24 hours treatment with $2.5 \mu \mathrm{M}$ DMAMQ. Both populations were gated identically using the unstained background control populations shown in grey behind the Ki67 negative (blue) and Ki67 positive (red) populations. (b) Quantification of the percentage increase in Ki67 expression from the profiles in $a$; Students $t$-test, $p=0.025, n=4$. (c) Quantification of $\beta$ galactosidase staining following three days incubation with $2.5 \mu \mathrm{M}$ DMAMQ, change is expressed relative to control cells; Students $t$-test, $n=4$. (d) Flow cytometry profiles of 
DNA content as an indication of cell cycle phase after 24 hours treatment with $2.5 \mu \mathrm{M}$ DMAMQ. Control and DMAMQ samples were gated identically based on the control distribution and phases are shown as G0/G1 in blue, $\mathrm{S}$ in red, G2/M in pale green and debris in dark green. (e) Quantification of the percentage cells in each phase of the cell cycle from the profiles in $d$; two-way ANOVA, $p<0.05, n=4$. (f) Quantification of the number of neurospheres grown in the colony forming assay with outgrowths in response to DMAMQ; Students $t$-test, $n=3$ ). (g) Quantification of the ability of differentiating NSCs to form neurite outgrowths in the presence of DMAMQ expressed relative to control cell outgrowths; Students $t$-test, $n=6 . * p<0.05, * * p<0.01$. 
(a) GFAP / NF-L / DAPI
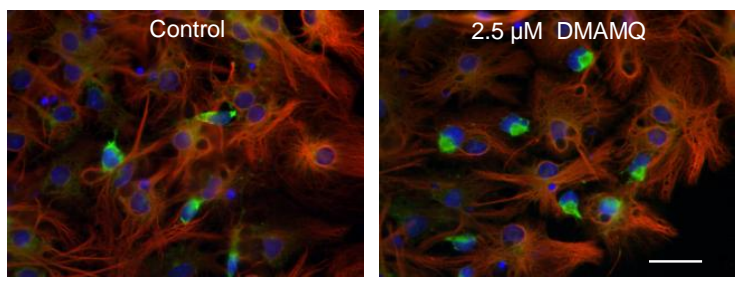

(b)
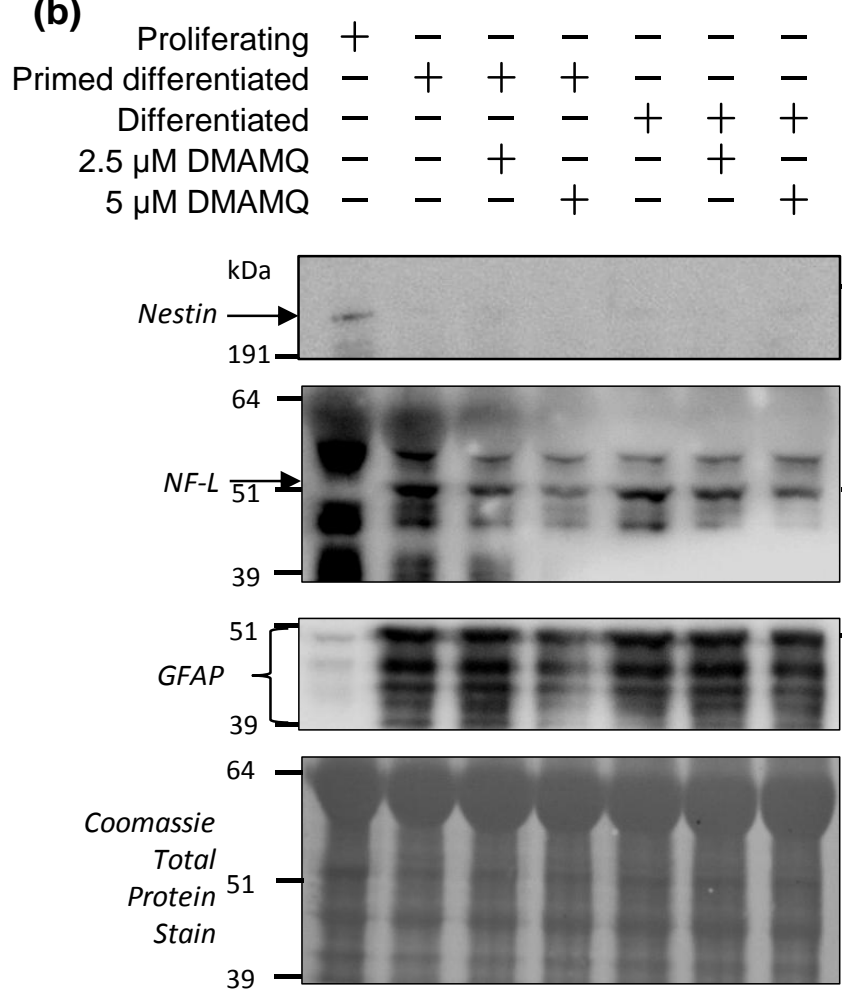
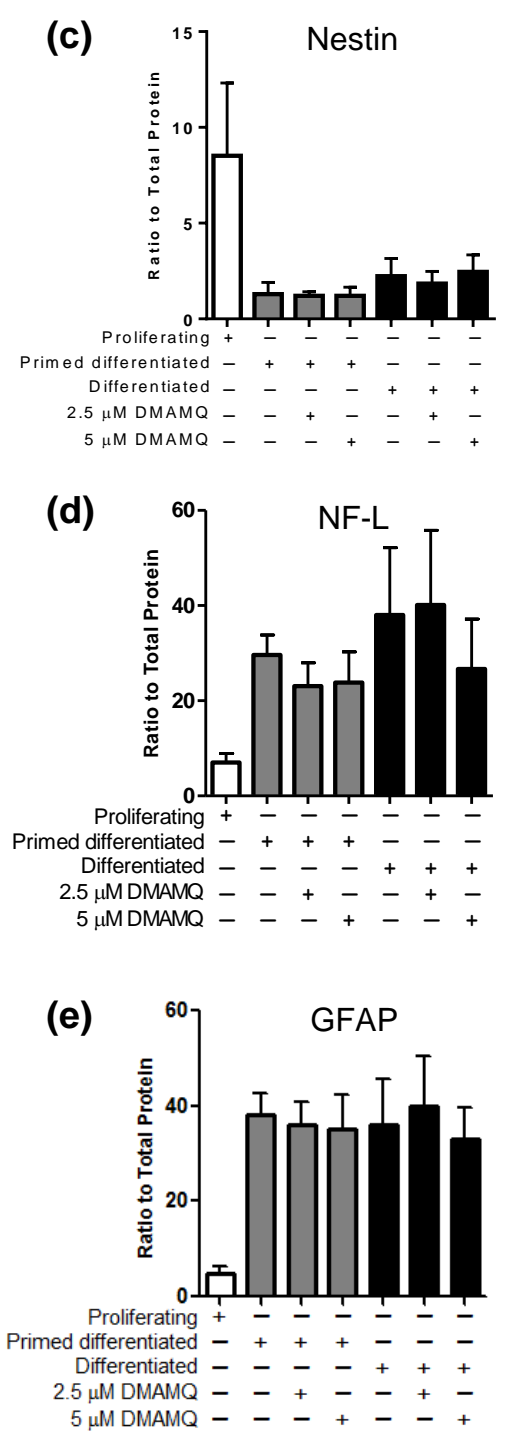

Figure 4. Effects of DMAMQ on NSC differentiation. (a) Immunofluorescence staining demonstrates that NSCs treated with DMAMQ throughout 7 days differentiation retain their capacity to differentiate and are multipotent. Green, NF-L (neuronal marker); Red, GFAP (astrocytic marker); Blue, DAPI (nuclear stain); Scale bar $=20 \mu \mathrm{m} . \quad$ (b) Example western blot images of cells either differentiated after 24 hours priming with DMAMQ or differentiated in the presence of DMAMQ. Densitometric quantification of western blot intensity for (c) nestin, (d) NF-L and (e) GFAP relative to the total-protein (coomassie) loading control. No differences are observed in lineage preference as a result of DMAMQ exposure either before or during differentiation; one-way ANOVA $n=5$. 
(a)
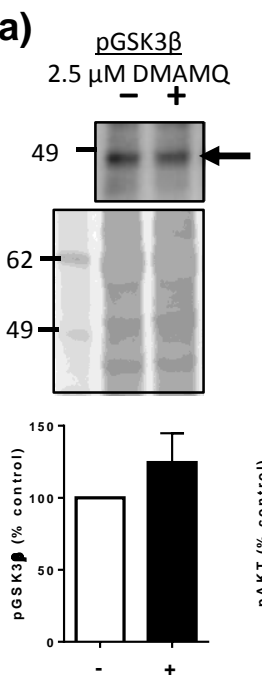
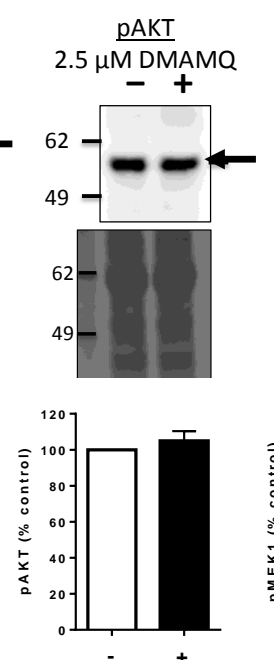

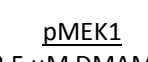

$2.5 \mu \mathrm{M}$ DMAMQ
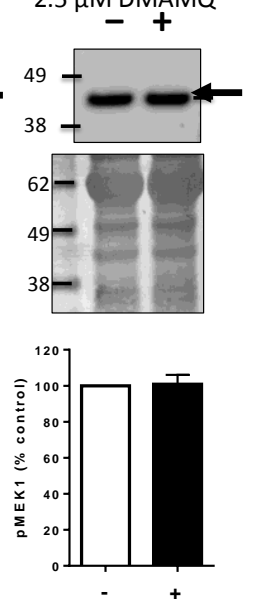

PMEK2
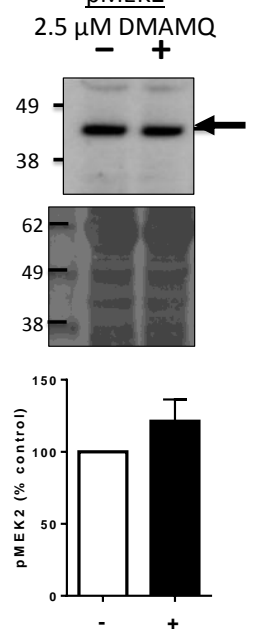

(b)

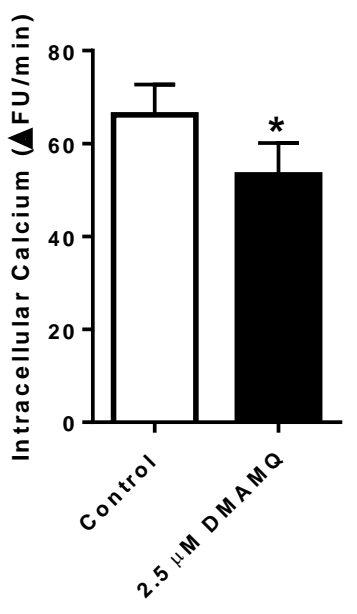

Figure 5. DMAMQ signalling is not through GSK-3 $\beta$, AKT or MEK signalling pathways. (a) NSCs were incubated for 30 minutes with DMAMQ and assessed for activation of signal transduction pathways by detection of changes in phosphorylation of several signalling intermediates (GSK-3 $\beta$, AKT, MEK1 and MEK2). No significant differences were seen in phosphorylation; Students $t$-test, $n=4$. (b) Intracellular calcium measured by fluorescence change per minute for 1 hour post DMAMQ addition. Students $t$-test, $n=4,{ }^{*} p<0.05$. 
(a)

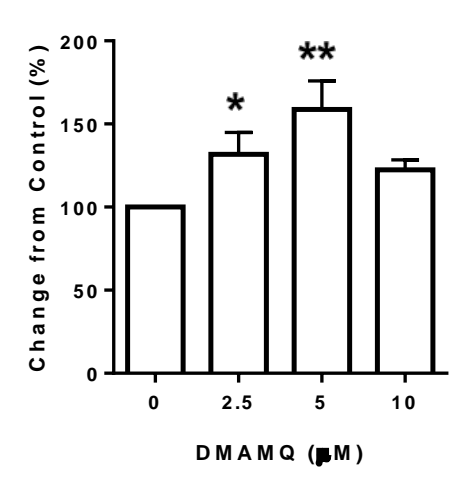

(b)

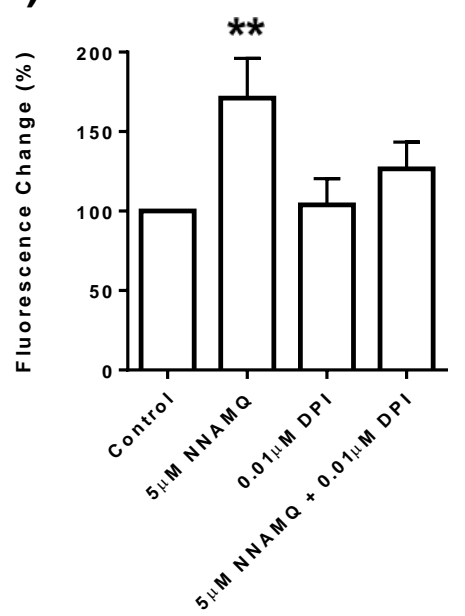

Figure 6. DMAMQ mediates its effects through stimulation of NADPH oxidase to increase intracellular ROS production. (a) Intracellular ROS increases in response to DMAMQ treatment in proliferating NSCs treated with DMAMQ in the low $\mu \mathrm{M}$ range, as measured by DCF fluorescence. (b) Co-treatment with DMAMQ and the NOX inhibitor, DPI, prevents the DMAMQ ROS burst, suggesting the ROS increase is associated with cellular signaling involving NADPH oxidase. One-way ANOVA, $n=4,{ }^{*} p<0.05, * * p<0.01$ (significantly different from untreated control). 
(a)
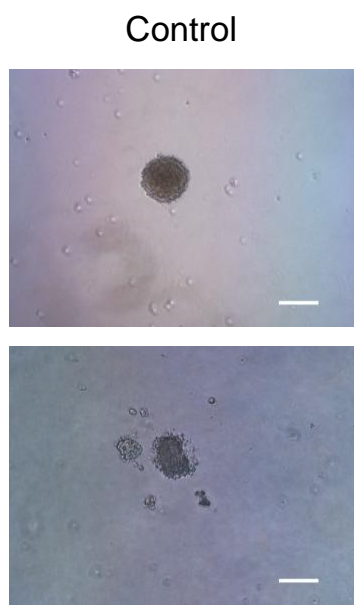

(b)

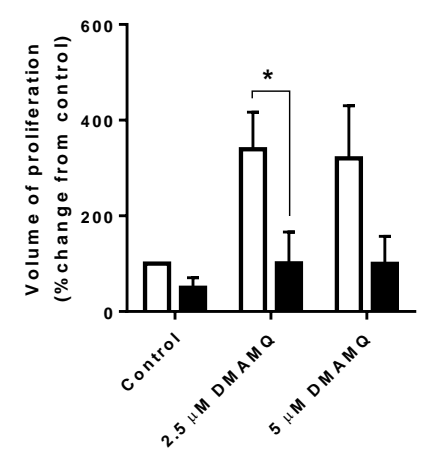

DMAMQ
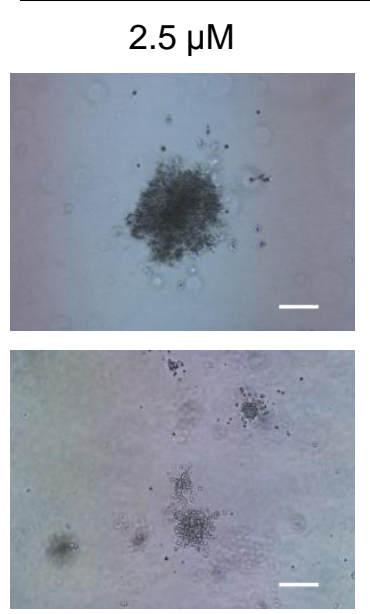

(c)

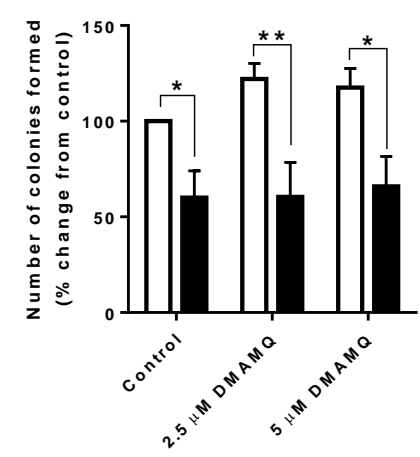

$5 \mu \mathrm{M}$

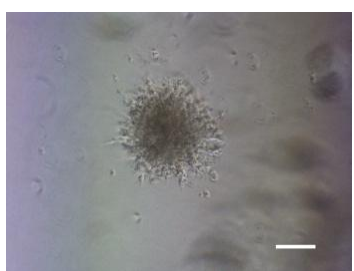

No treatment

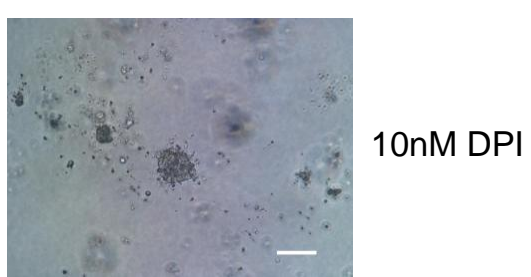

(d)

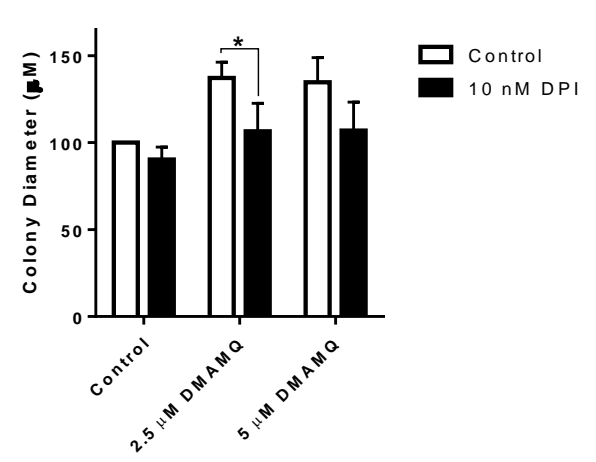

Figure 7. Nox inhibition with DPI blocks DMAMQ induced growth. (a) Representative colonys grown in the presence or absence of Nox family inhibitor DPI and DMAMQ. Scale bar, $100 \mu \mathrm{m}$. (b) Total volume of growth at 21 days calculated from the number of colonies formed (c) and the diameter of the colonies (d). Two-way ANOVA, $n=3, * p<0.05$, **p $<$ 0.01 . 


\section{References}

1. Gomez-Nicola D, Suzzi S, Vargas-Caballero M, Fransen NL, Al-Malki H, Cebrian-Silla A, Garcia-Verdugo JM, Riecken K, Fehse B, Perry VH (2014) Temporal dynamics of hippocampal neurogenesis in chronic neurodegeneration. Brain 137, 2312-2328.

2. Jin K, Peel AL, Mao XO, Xie L, Cottrell BA, Henshall DC, Greenberg DA (2004) Increased hippocampal neurogenesis in Alzheimer's disease. Proc. Natl. Acad. Sci. USA 101, 343-347.

3. Haughey NJ, Nath A, Chan SL, Borchard AC, Rao MS, Mattson MP (2002) Disruption of neurogenesis by amyloid beta-peptide, and perturbed neural progenitor cell homeostasis, in models of Alzheimer's disease. J. Neurochem. 83, 1509-1524.

4. Jin K, Galvan V, Xie L, Mao XO, Gorostiza OF, Bredesen DE, Greenberg DA (2004) Enhanced neurogenesis in Alzheimer's disease transgenic (PDGF-APPSw,Ind) mice. Proc. Natl. Acad. Sci. USA 101, 13363-13367.

5. Kanemoto S, Griffin J, Markham-Coultes K, Aubert I, Tandon A, George-Hyslop PS, Fraser PE (2014) Proliferation, differentiation and amyloid-beta production in neural progenitor cells isolated from TgCRND8 mice. Neuroscience 261, 52-59.

6. Karkkainen V, Magga J, Koistinaho J, Malm T (2012) Brain environment and Alzheimer's disease mutations affect the survival, migration and differentiation of neural progenitor cells. Curr. Alzheimer Res. 9, 1030-1042.

7. Lopez-Toledano MA, Shelanski ML (2007) Increased neurogenesis in young transgenic mice overexpressing human APP(Sw, Ind). J. Alzheimers Dis. 12, 229-240.

8. Akers KG, Martinez-Canabal A, Restivo L, Yiu AP, De Cristofaro A, Hsiang HL, Wheeler AL, Guskjolen A, Niibori Y, Shoji H, Ohira K, Richards BA, Miyakawa T, Josselyn SA, 
Frankland PW (2014) Hippocampal neurogenesis regulates forgetting during adulthood and infancy. Science 344, 598-602.

9. Dupret D, Revest JM, Koehl M, Ichas F, De Giorgi F, Costet P, Abrous DN, Piazza PV (2008) Spatial relational memory requires hippocampal adult neurogenesis. PLoS ONE 3, e1959.

10. Sahay A, Scobie KN, Hill AS, O'Carroll CM, Kheirbek MA, Burghardt NS, Fenton AA, Dranovsky A, Hen R (2011) Increasing adult hippocampal neurogenesis is sufficient to improve pattern separation. Nature 472, 466-470.

11. Leifer W, Steiner K (1949) Diodoquin as a topical therapeutic agent in cutaneous. J. Invest. Dermatol. 12, 203-204.

12. Gershon H, Gershon M, Clarke DD (2011) Antifungal activity of substituted 8-quinolinol5- and 7-sulfonic acids: a mechanism of action is suggested based on intramolecular synergism. Mycopathologia 155, 213-217.

13. Zhou J, Zhang H, Gu P, Margolick JB, Yin D, Zhang Y (2009) Cancer stem/progenitor cell active compound 8-quinolinol in combination with paclitaxel achieves an improved cure of breast cancer in the mouse model. Breast Cancer Res. Treat. 115, 269-277.

14. Mohammed I, Hampton SE, Ashall L, Hildebrandt ER, Kutlik RA, Manandhar SP, Floyd BJ, Smith HE, Dozier JK, Distefano MD, Schmidt WK, Dore TM (2016) 8Hydroxyquinoline-based inhibitors of the Rce1 protease disrupt Ras membrane localization in human cells. Bioorg. Med. Chem. 24, 160-178.

15. Martirosyan A1, Leonard S, Shi X, Griffith B, Gannett P, Strobl J (2006) Actions of a histone deacetylase inhibitor NSC3852 (5-nitroso-8-quinolinol) link reactive oxygen species 
to cell differentiation and apoptosis in MCF-7 human mammary tumor cells. J. Pharmacol. Exp. Ther. 317, 546-552.

16. King ONF, Li XS, Sakurai M, Kawamura A, Rose NR, Ng SS, Quinn AM, Rai G, Mott BT, Beswick P, Klose RJ, Oppermann U, Jadhav A, Heightman TD, Maloney DJ, Schofield CJ, Simeonov A (2011) Quantitative High-Throughput Screening Identifies 8Hydroxyquinolines as Cell-Active Histone Demethylase Inhibitors. PLoS ONE 5, e15535.

17. Tardiff DF, Tucci ML, Caldwell KA, Caldwell GA, Lindquist S (2011) Different 8hydroxyquinolines protect models of TDP-43 protein, $\alpha$-synuclein, and polyglutamine proteotoxicity through distinct mechanisms. J. Biol. Chem. 287, 4107-4120.

18. Crouch PJ, Savva MS, Hung LW, Donnelly PS, Mot AI, Parker SJ, Greenough MA, Volitakis I, Adlard PA, Cherny RA, Masters CL, Bush AI, Barnham KJ, White AR (2011) The Alzheimer's therapeutic PBT2 promotes amyloid- $\beta$ degradation and GSK3 phosphorylation via a metal chaperone activity. J. Neurochem. 119, 220-230.

19. Adlard PA, Bica L, White AR, Nurjono M, Filiz G, Crouch PJ, Donnelly PS, Cappai R, Finkelstein DI, Bush AI (2011) Metal Ionophore Treatment Restores Dendritic Spine Density and Synaptic Protein Levels in a Mouse Model of Alzheimer's Disease. PLoS ONE 6, e17669.

20. Gomes LM, Vieira RP, Jones MR, Wang MC, Dyrager C, Souza-Fagundes EM, Da Silva JG, Storr T, Beraldo H (2014) 8-Hydroxyquinoline Schiff-base compounds as antioxidants and modulators of copper-mediated A $\beta$ peptide aggregation. J. Inorg. Biochem. 139, 106116.

21. Mancin F, Chin J (2002) An artificial guanine that binds cytidine through the cooperative interaction of metal coordination and hydrogen bonding. J. Am. Chem. Soc. 124, 1094610947. 
22. Johnson DK, Kline SJ (1991) 8-hydroxyquinoline chelating agents. United States Patent No. 5021567 A, 4 June.

23. Rajagopalan R, Archilefu SI, Bugaj JE, Dorshow RB (2011) Quinoline Ligands and Metal Complexes for Diagnosis and Therapy. United States Patent No. 6277841 B1, Aug. 21.

24. Schwarcz R, Bruno JP, Muchowski PJ, Wu HQ (2012) Kynurenines in the mammalian brain: when physiology meets pathology. Nat. Rev. Neurosci. 13, 465-477.

25. Mossialos D, Meyer J-M, Budzikiewicz H, Wolff U, Koedam N, Baysse C, Anjaiah V, Cornelis P (2000) Quinolobactin, a New Siderophore of Pseudomonas fluorescens ATCC 17400, the Production of Which Is Repressed by the Cognate Pyoverdine. Appl. Environ. Microbiol. 66, 487-492.

26. Pesek J, Svoboda J, Sattler M, Bartram S, Boland W (2015) Org Biomol Chem. 13, 178184.

27. Kenche VB, Zawisza I, Masters CL, Bal W, Barnham KJ, Drew SC (2013) Mixed Ligand $\mathrm{Cu}^{2+}$ Complexes of a Model Therapeutic with Alzheimer's Amyloid- $\beta$ Peptide and monoamine Neurotransmitters. Inorg. Chem. 52, 4303-4318.

28. Haigh CL, McGlade AR, Lewis V, Masters CL, Lawson VA, Collins SJ (2011) Acute exposure to prion infection induces transient oxidative stress progressing to be cumulatively deleterious with chronic propagation in vitro. Free Radic. Biol. Med. 2011, 51, 594-608.

29. Collins SJ, Tumpach C, Li Q-X, Lewis V, Ryan TM, Roberts B, Drew SC, Lawson VA, Haigh CL (2015) The prion protein regulates $\beta$-amyloid mediated self-renewal of neural stem cells. Stem Cell Res. Therap. 6:60. 
30. Haigh CL, Drew SC, Boland M, Masters CL, Barnham KJ, Lawson VA, Collins SJ (2009) Dominant roles of the polybasic proline motif and copper in PrP23-89 mediated stress protection response. J. Cell. Sci.122, 1518-1528.

31. Sinclair L, Lewis V, Collins SJ, Haigh CL (2013) Cytosolic caspases mediate mislocalised SOD2 depletion in an in vitro model of chronic prion infection. Dis. Model Mech. 6, 952-963.

32. Haigh CL, Lewis VA, Vella LJ, Masters CL, Hill AF, Lawson VA, Collins SJ (2009) PrPC-related signal transduction is influenced by copper, membrane integrity and the alpha cleavage site. Cell Res. 19, 1062-1078.

33. Lang C, Mix E, Frahm J, Glass Ä, Müller J, Schmitt O, Schmöle A-C, Klemm K, Ortinau S, Hübner R, Frecha MJ, Wree A, Rolfs A (2011) Small molecule GSK-3 inhibitors increase neurogenesis of human neural progenitor cells. Neurosci. Lett. 488, 36-40.

34. Morales-Garcia JA, Luna-Medina R, Alonso-Gil S, Sanz-Sancristobal M, Palomo V, Gil C, Santos A, Martinez A, Perez-Castillo A (2012) Glycogen synthase kinase 3 inhibition promotes adult hippocampal neurogenesis in vitro and in vivo. ACS Chem. Neurosci. 3, 963971.

35. Tonelli FM, Santos AK, Gomes DA, da Silva SL, Gomes KN, Ladeira LO, Resende RR (2012) Stem cells and calcium signalling. Adv. Exp. Med. Biol. 740, 891-916.

36. Bogeski I, Kappl R, Kummerow C, Gulaboski R, Hoth M, Niemeyer BA (2011) Redox regulation of calcium ion channels: Chemical and physiological aspects. Cell Calcium 50, $407-423$.

37. Panday A, Sahoo MK, Osorio D, Batra A (2015) NADPH oxidases: an overview from structure to innate immunity-associated pathologies. Cell. Mol. Immunol. 12, 5-23. 
38. Le Belle JE, Orozco NM, Paucar AA, Saxe JP, Mottahedeh J, Pyle AD, Wu H, Kornblum HI (2011) Proliferative neural stem cells have high endogenous ROS levels that regulate selfrenewal and neurogenesis in a PI3K/Akt-dependant manner. Cell Stem Cell 8, 59-71.

39. Walton NM, Shin R, Tajinda K, Heusner CL, Kogan JH, Miyake S, Chen Q, Tamura K, Matsumoto M (2012) Adult neurogenesis transiently generates oxidative stress. PLoS ONE, 7, e35264.

40. Yoneyama M, Kawada K, Gotoh Y, Shiba T, Ogita K (2010) Endogenous reactive oxygen species are essential for proliferation of neural stem/progenitor cells. Neurochem. Int. 56, $740-746$.

41. Menon SG, Goswami PC (2007) A redox cycle within the cell cycle: ring in the old with the new. Oncogene 26, 1101-1109.

42. Sarsour EH, Kalen AL, Goswami PC (2014) Manganese superoxide dismutase regulates a redox cycle within the cell cycle. Antioxid. Redox Signal. 20, 1618-1627.

43. Lee JE, Cho KE, Lee KE, Kim J, Bae YS (2014) Nox4-mediated cell signaling regulates differentiation and survival of neural crest stem cells. Mol. Cells 37, 907-911.

44. Nayernia Z, Jaquet V, Krause KH (2014) New insights on NOX enzymes in the central nervous system. Antioxid. Redox Signal. 20, 2815-2837.

45. Topchiy E, Panzhinskiy E, Griffin WS, Barger SW, Das M, Zawada WM (2013) Nox4generated superoxide drives angiotensin II-induced neural stem cell proliferation. Dev. Neurosci. 35, 293-305.

46. Barnham KJ, Masters CL, Bush AI (2004) Neurodegenerative diseases and oxidative stress. Nat. Rev. Drug Discov. 3, 205-214. 
47. Zheng M, Liu J, Ruan Z, Tian S, Ma Y, Zhu J, Li G (2013) Intrahippocampal injection of Abeta1-42 inhibits neurogenesis and down-regulates IFN-gamma and NF-kappaB expression in hippocampus of adult mouse brain. Amyloid 20, 13-20.

48. Haughey NJ, Liu D, Nath A, Borchard AC, Mattson MP (2002) Disruption of neurogenesis in the subventricular zone of adult mice, and in human cortical neuronal precursor cells in culture, by amyloid beta-peptide: implications for the pathogenesis of Alzheimer's disease. Neuromolecular Med. 1, 125-135.

49. M. Díaz-Moreno M, Hortigüela R, Gonçalves A, García-Carpio I, Manich G, GarcíaBermúdez E, Moreno-Estellés M, Eguiluz C, Vilaplana J, Pelegrí C, Vilar M, Mira H (2013) A $\beta$ increases neural stem cell activity in senescence-accelerated SAMP8 mice. Neurobiol. Aging 34, 2623-2638.

50. Gonzalez DR, Treuer AV, Lamirault G, Mayo V, Cao Y, Dulce RA, Hare JM (2014) NADPH oxidase-2 inhibition restores contractility and intracellular calcium handling and reduces arrhythmogenicity in dystrophic cardiomyopathy. Am. J. Physiol. Heart Circ. Physiol. 307, H710-H721.

51. Zhang M, Prosser BL, Bamboye MA, Gondim AN, Santos CX, Martin D, Ghigo A, Perino A, Brewer AC, Ward CW, Hirsch E, Lederer WJ, Shah AM (2015) Contractile Function During Angiotensin-II Activation: Increased Nox2 Activity Modulates Cardiac Calcium Handling via Phospholamban Phosphorylation. J. Am. Coll. Cardiol. 66, 261-272.

52. Rada B, Leto TL (2008) Oxidative innate immune defenses by Nox/Duox family NADPH Oxidases. Contrib. Microbiol. 15, 164-187.

53. Cooney SJ, Bermudez-Sabogal SL, Byrnes KR (2013) Cellular and temporal expression of NADPH oxidase (NOX) isotypes after brain injury. J. Neuroinflammation.10:155. 
Cellular and temporal expression of NADPH oxidase (NOX) isotypes after brain injury.

54. Fukui T, Asakura K, Hikichi C, Ishikawa T, Murai R, Hirota S, Murate K, Kizawa M, Ueda A, Ito S, Mutoh T (2015) Histone deacetylase inhibitor attenuates neurotoxicity of clioquinol in PC12 cells. Toxicology 331, 112-118.

55. Sampson EL, Jenagaratnam L, McShane R (2014) Metal protein attenuating compounds for the treatment of Alzheimer's dementia. Cochrane Database Syst. Rev. 2, CD005380. 
